\title{
Sound Radiation of a Pulsating Sphere in the Outlet of a Hard/Soft Semi-Spherical Cavity in a Flat Screen
}

\author{
Wojciech P. RDZANEK \\ Department of Mechatronics and Control Science \\ Faculty of Mathematics and Natural Sciences \\ University of Rzeszów \\ Prof. St. Pigonia 1, 35-310 Rzeszów, Poland; e-mail: wprdzank@gmail.com,wprdzank@ur.edu.pl
}

(received July 29, 2015; accepted October 21, 2015)

\begin{abstract}
A rigorous analysis of sound radiation by a pulsating sphere forming a resonator together with a semispherical cavity is presented. Both hard and soft boundaries are considered, as well as mixed. The problem is solved by dividing the entire region into two subregions, one surrounding the sphere and containing the cavity and the other for the remaining half-space. The continuity conditions are applied to obtain the acoustic pressure. Then the acoustic radiation resistance is calculated both in the near- and far-field. The acoustic radiation reactance is calculated in the impedance approach. The resonance frequencies are determined, for which a significant growth of the sound pressure level is observed as well as the sound field directivity. The accuracy and convergence of these rigorous results has been examined empirically.
\end{abstract}

Keywords: Neumann/Dirichlet problem; sound field; acoustic impedance; continuity.

\section{Introduction}

The radiation and scattering of acoustic waves by spherical objects are important in an oceanic environment. The results of scientific investigations are usually useful for designing the depth sounders and sonar antennas applied in fishery, water warfare, biology and medicine. The results obtained are often useful in different branches of science. So far, there have been a number of studies dealing with this topic. FOLDY (1949) presented the power efficiency of passive linear electroacoustic transducers. ANDERson (1950) analyzed sound scattering by a spherical obstacle of size comparable to the wavelength. FARAN (1951) considered sound scattering by cylindrical and spherical obstacles containing shear waves in addition to compressional waves. FERRIS (1952) analyzed the natural frequencies and mode shapes of a spherical cavity filled with a gaseous medium. RudGers (1974) studied the acoustic impedance of an array of complex transducers in a cylindrical baffle with spherical caps at both of its ends with an additional cylindrical reflector considering the reciprocal interactions of the transducers in detail. THOMPSON JR. $(1973 ; 1976)$ considered an interesting problem of sound radiation by a sphere eccentrically located within a fluid sphere using the Clebsch-Gordan coefficients, which enables expressing the acoustic field of a spherical source in some eccentric spherical coordinates, and also solved the problem of sound radiation by a spherical source located near a fluid sphere. The method was further developed by GAUNAURD and HUANG (1996), who used it for solving the problem of sound scattering by a spherical object near a hard flat bottom. HASHEMINEJAD (2003) analyzed rigorously the modal radiation load on a sphere immersed in an acoustic halfspace bounded by a rigid plane using the same method. HASHEMINEJAD and AzARPEYVAND (2003a,b; 2004a,b) used the method for solving the problem of the asymmetric sound radiation by an oscillating sphere above a rigid/compliant plane. They also investigated the problem of sound radiation by a spherical cap near a hard/soft flat screen and the sound radiation from a vibrating sphere within an acoustic quarterspace. Another achievement of theirs is describing in detail the acoustic radiation from a sphere suspended within a thermoviscous fluid sphere, including the effects of eccentricity. AzARPEYVAND and AzARPEYVAND (2014) analyzed theoretically the acoustic manipulation of porous spherical shells for applications in magnetic resonance imaging using high order Bessel beams and Biot's theory of poro-elasticity. LEvine and LEPPINGTON (1991) considered analytically the acoustic power from moving and pulsating spheres. LEvine (2001) presented a tu- 
torial exposition of cavity excitations with some simple analytical methods. BRAŃSKI and LENIOWsKA (1992) investigated the far field of a concentric ring vibrating on a rigid sphere over a side branch deep cavity in a rectangular duct. AARTS and JANSSEN (2010; 2011) used the Zernike polynomials to model the sound radiation from a resilient spherical cap on a rigid sphere. TANG, WU and TANG (2010) improved the sound radiation performance of a spherical cap radiator by adding an acoustic soft material belt between the vibrating cap and the rigid baffle. BARMATZ and COLLAS (1985) studied the acoustic potential on a sphere for small wavelengths. KIM, LAUCHLE, and GABRIELSON (2008) performed measurements of the near-field acoustic intensity under water. AzARPEYVAND (2005; 2014) created silent zones in a diffuse sound field using a vibrating spherical piston, and also analyzed the phenomenon of backward dragging forces on objects illuminated by a symmetric acoustic Bessel beam. The sound radiation was also examined in different boundary geometries. PELAT, FÉLIX and PAGneux (2009) presented the use of leaky modes in open waveguides for the sound propagation modeling in street canyons. SzEMELA (2015) examined sound radiation of vibrating piston in an acoustic canyon with walls of infinite height. Kolber, SnAkowska, and Kozupa (2014) studied the effect of plate discretization on accuracy of the sound radiation efficiency measurements for a speaker embedded into a rectangular cavity. JEONG, KANG and KIM (2012) presented an analytical model for sound radiation of a Korean bell.

Although the literature on the acoustic field of a spherical source is extensive, the problem of the sound radiation of a pulsating rigid sphere located in the outlet of a hemispherical baffle in a flat screen for four different boundary configurations of the baffle has not been treated using rigorous methods, as far as the author's knowledge extends. This problem is, therefore, treated in this paper.

\section{The acoustic field}

The Neumann/Dirichlet boundary problem is considered for a pulsating rigid sphere of radius $a$. The sphere is embedded in the outlet of a semi-spherical cavity of radius $b$ in screen (cf. Fig. 1). The problem is axially symmetric. The Helmholtz equation

$$
\left(\nabla^{2}+k^{2}\right) p(r, \theta, t)=0
$$

is satisfied within the entire region $\Omega$ filled with water, where the acoustic pressure

$$
p(r, \theta, t)=p(r, \theta) \exp (-\mathrm{i} \omega t)
$$

is time harmonic, $p(r, \theta)$ is the amplitude, $\exp (-\mathrm{i} \omega t)$ is the time dependence (which will be suppressed in the further analysis), $\omega$ is the angular frequency, $k$ is

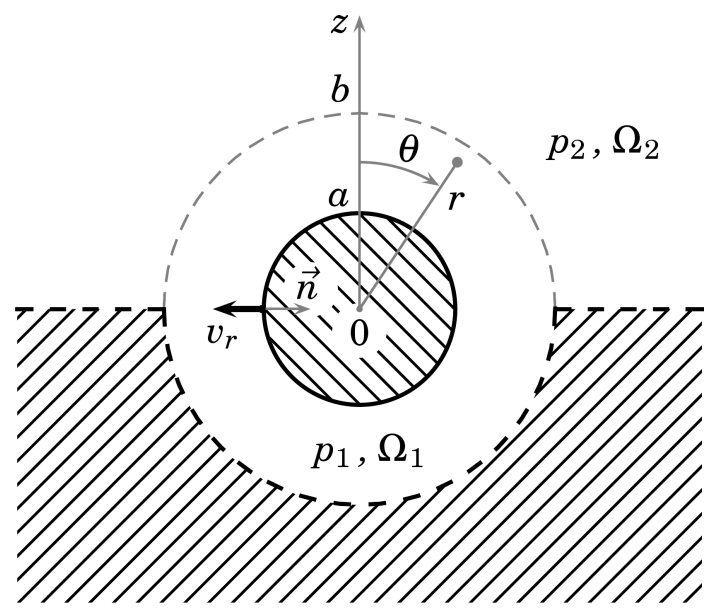

Fig. 1. The pulsating rigid sphere of radius $a$ in the outlet of the hard/soft semi-spherical cavity (the region $\Omega=\Omega_{1}+\Omega_{2}$ ).

the wavenumber, and $(r, \theta)$ are spherical coordinates. Assuming that the sphere is perfectly rigid, and that the surfaces of the cavity and the screen can be either acoustically hard or soft, it is possible to distinguish four different boundary problems, as illustrated in Fig. 2 two with homogeneous boundaries, hard or soft, and the other two with mixed boundaries, hardsoft or soft-hard. Since all the problems are solved similarly, only one of them will be presented in detail. For the other three problems, the main differences will be presented. For this purpose, the region $\Omega=\Omega_{1}+\Omega_{2}$ of the semi-spherical cavity and the half-space has been artificially divided into two subregions (cf. Fig. 1)

$$
\begin{aligned}
& \Omega_{1}=\{0<a \leqslant r \leqslant b ; 0 \leqslant \theta \leqslant \pi\}, \\
& \Omega_{2}=\{b \leqslant r \leqslant \infty ; 0 \leqslant \theta \leqslant \pi / 2\} .
\end{aligned}
$$

The region $\Omega_{1}$ is the annular spherical layer, and the region $\Omega_{2}$ covers the rest of the considered space. The acoustic pressures has been denoted by $p_{1}$ and $p_{2}$ for the two subregions. a)

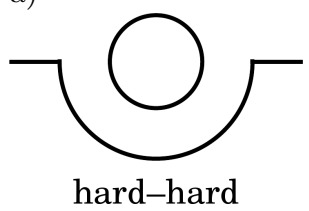

c)

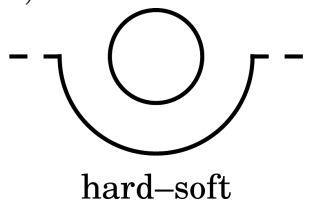

b)

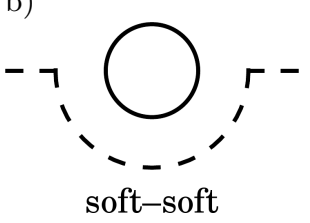

d)

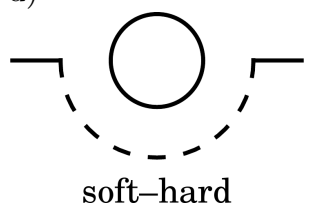

Fig. 2. Four different boundary configurations. 


\subsection{An acoustically hard boundary}

In the case of an acoustically hard boundary (cf. Fig. 2a), the following boundary conditions are satisfied

$$
\begin{aligned}
& \left.\frac{\partial p_{1}}{\partial r}\right|_{r=a}=-\mathrm{i} k \varrho_{0} c v_{0} ; \quad 0 \leqslant \theta \leqslant \pi, \\
& p_{1}(b, \theta)=p_{2}(b, \theta) ; \quad 0 \leqslant \theta \leqslant \pi / 2, \\
& \left.\frac{\partial p_{1}}{\partial r}\right|_{r=b}=\left\{\begin{array}{cc}
\left.\frac{\partial p_{2}}{\partial r}\right|_{r=b} ; & 0 \leqslant \theta \leqslant \pi / 2, \\
0 ; & \pi / 2 \leqslant \theta \leqslant \pi,
\end{array}\right. \\
& \left.\frac{\partial p_{2}}{\partial \theta}\right|_{\theta=\pi / 2}=0 ; \quad b \leqslant r \leqslant \infty
\end{aligned}
$$

where $\varrho_{0}$ is the rest density of water and $c$ is the speed of sound. The radiation condition (SOMMERFELD, 1964)

$$
\lim _{r \rightarrow \infty} r\left(\frac{\partial p_{2}}{\partial r}-\mathrm{i} k p_{2}\right)=0
$$

is also satisfied. The normal component of the vibrational velocity on the sphere for $r=a$ (the normal unit vector $\vec{n}$ is oriented outside the considered region $\Omega$ ) can be formulated as

$$
v(t)=v_{0} \exp (-\mathrm{i} \omega t)
$$

for the steady time harmonic vibrations, where $v_{0} \in \mathbb{C}$ is the amplitude and $S=4 \pi a^{2}$ is the area of pulsating sphere. The acoustic pressure amplitude (referred to in the future as the acoustic pressure) can be expressed as (Morse, 1948, Eq. 27.13, pg. 317)

$$
\begin{array}{lr}
p_{1}(r, \theta)=\sum_{n=0}^{\infty}\left[A_{n} j_{n}(k r)+B_{n} h_{n}^{(1)}(k r)\right] P_{n}(\cos \theta) & \text { for } \Omega_{1} \\
p_{2}(r, \theta)=\sum_{n=0}^{\infty} C_{n} h_{2 n}^{(1)}(k r) P_{2 n}(\cos \theta) ; & \text { for } \Omega_{2}
\end{array}
$$

where $j_{n}$ and $h_{n}^{(1)}$ are the spherical Bessel and Hankel functions of the $n$-th order, and the index values $2 n$ result from the condition $\left.(\mathrm{d} / \mathrm{d} \theta) P_{2 n}(\cos \theta)\right|_{\theta=\pi / 2}=0$ for $r \geqslant b$. The amplitude coefficients $B_{n}$ can be expressed by applying the boundary condition in Eq. (4) to Eq. $(7)_{1}$, multiplying the latter side by side by $P_{n^{\prime}}(\cos \theta) \sin \theta$, integrating it with respect to $\theta$ over the interval from 0 to $\pi$, using the orthogonality of the Legendre polynomials in Eq. $(2)_{1}$, which gives $B_{n}=$ $-\left\{\mathrm{i} \varrho_{0} c v_{0} h_{0}^{(1)}(k r)+A_{n} j_{n}^{\prime}(k a)\right\}\left[h_{0}^{(1) '}(k a)\right]^{-1}$. Then inserting it into Eq. $(7)_{1}$ yields

$$
\begin{aligned}
p_{1}(r, \theta)= & -\mathrm{i} \varrho_{0} c v_{0} \frac{h_{0}^{(1)}(k r)}{h_{0}^{(1) \prime}(k a)} \\
& +\sum_{n=0}^{\infty} A_{n} z_{n}^{(1)}(k r, k a) P_{n}(\cos \theta)
\end{aligned}
$$

for $\Omega_{1}$, where

$$
z_{n}^{(1)}(k r, k a)=j_{n}(k r)-\frac{j_{n}^{\prime}(k a)}{h_{n}^{(1) \prime}(k a)} h_{n}^{(1)}(k r) .
$$

Further, we insert the solution in Eqs. $(7)_{2}$ and (8) into the boundary condition $(4)_{2}$, multiply side by side by $P_{2 n^{\prime}}(\cos \theta) \sin \theta$, integrate with respect to $\theta$ from 0 to $\pi / 2$, use the orthogonality condition in Eq. $(38)_{2}$ and $(38)_{3}$, and obtain

$$
\begin{aligned}
A_{2 n^{\prime}} z_{2 n^{\prime}}^{(1)}(k b, k a)+ & N_{2 n^{\prime}} \sum_{n=0}^{\infty} A_{2 n+1} z_{2 n+1}^{(1)}(k b, k a) \\
& \cdot \bar{P}_{2 n^{\prime}, 2 n+1}-C_{n^{\prime}} h_{2 n^{\prime}}^{(1)}(k b) \\
= & \delta_{0, n^{\prime}} \mathrm{i} \varrho_{0} c v_{0} \frac{h_{0}^{(1)}(k b)}{h_{0}^{(1) \prime}(k a)},
\end{aligned}
$$

where the normalization constant is $N_{\nu}$ is presented in Eq. $(39)_{1}$, and $\bar{P}$ in Eqs. $(38)_{3}$ and $(38)_{2}$. Then we insert Eqs. $(7)_{2}$ and (8) into Eq. $(4)_{c}$, multiply side by side by $P_{n^{\prime}}(\cos \theta) \sin \theta$, integrate with respect to $\theta$ from 0 to $\pi$, use Eq. $(38)_{1}$, and obtain separately for even and odd values of $n^{\prime}$,

$$
\begin{gathered}
A_{2 n^{\prime}} z_{2 n^{\prime}}^{(1) \prime}(k b, k a)-\frac{1}{2} C_{n^{\prime}} h_{2 n^{\prime}}^{(1) \prime}(k b) \\
=\delta_{0, n^{\prime}} \mathrm{i} \varrho_{0} c v_{0} \frac{h_{0}^{(1) \prime}(k b)}{h_{0}^{(1) \prime}(k a)}, \\
A_{2 n^{\prime}+1} z_{2 n^{\prime}+1}^{(1) \prime}(k b, k a)-\frac{1}{2} N_{2 n^{\prime}+1} \sum_{n=0}^{\infty} C_{n} h_{2 n}^{(1) \prime}(k b) \\
\cdot \bar{P}_{2 n, 2 n^{\prime}+1}=0 .
\end{gathered}
$$

Equations (10) and (11) form the system of algebraic equations. Solving it numerically gives the unknown coefficients $A_{n}$ and $C_{n}$.

\subsection{Acoustically soft boundaries}

In the case of acoustically soft boundaries (cf. Fig. 2b), the boundary condition $(4)_{1}$ is satisfied as well as the set

$$
\begin{aligned}
p_{1}(b, \theta) & =\left\{\begin{array}{cc}
p_{2}(b, \theta) ; & 0 \leqslant \theta \leqslant \pi / 2, \\
0 ; & \pi / 2 \leqslant \theta \leqslant \pi,
\end{array}\right. \\
\left.\frac{\partial p_{1}}{\partial r}\right|_{r=b}=\left.\frac{\partial p_{2}}{\partial r}\right|_{r=b} ; & 0 \leqslant \theta \leqslant \pi / 2 \\
p_{2}(r, \pi / 2)=0 ; & b \leqslant r \leqslant \infty,
\end{aligned}
$$


along with the radiation condition (5). The acoustic pressure can be expressed using Eq. (8), and

$$
p_{2}(r, \theta)=\sum_{n=0}^{\infty} C_{n} h_{2 n+1}^{(1)}(k r) P_{2 n+1}(\cos \theta)
$$

for $\Omega_{2}$, where the index values $2 n+1$ result from the condition $P_{2 n+1}(0)=0$ for $r \geqslant b$. Inserting Eqs. (8) and (13 into $(12)_{1}$ and $(12)_{2}$, and processing in a similar way as in the former case, finally gives

$$
\begin{gathered}
A_{2 n^{\prime}} z_{2 n^{\prime}}^{(1)}(k b, k a)-\frac{1}{2} N_{2 n^{\prime}} \sum_{n=0}^{\infty} C_{n} h_{2 n+1}^{(1)}(k b) \bar{P}_{2 n^{\prime}, 2 n+1} \\
=\delta_{0, n^{\prime}} \mathrm{i} \varrho_{0} c v_{0} \frac{h_{0}^{(1)}(k b)}{h_{0}^{(1) \prime}(k a)}, \\
A_{2 n^{\prime}+1} z_{2 n^{\prime}+1}^{(1)}(k b, k a)-\frac{1}{2} C_{n^{\prime}} h_{2 n^{\prime}+1}^{(1)}(k b)=0 \\
N_{2 n^{\prime}+1} \sum_{n=0}^{\infty} A_{2 n} z_{2 n}^{(1) \prime}(k b, k a) \bar{P}_{2 n, 2 n^{\prime}+1} \\
+A_{2 n^{\prime}+1} z_{2 n^{\prime}+1}^{(1) \prime}(k b, k a)-C_{n^{\prime}} h_{2 n^{\prime}+1}^{(1) \prime}(k b) \\
=\mathrm{i} \varrho_{0} c v_{0} N_{2 n^{\prime}+1} \bar{P}_{0,2 n^{\prime}+1} \frac{h_{0}^{(1) \prime}(k b)}{h_{0}^{(1) \prime}(k a)}
\end{gathered}
$$

Solving numerically the above system of algebraic equations gives $A_{n}$ and $C_{n}$.

\subsection{Acoustically hard-soft boundaries}

In the case of acoustically hard-soft mixed boundaries (cf. Fig. 2c), the boundary conditions $(4)_{1},(4)_{2}$, $(4)_{3}$, and $(12)_{3}$ are satisfied along with the radiation condition (5). Inserting Eqs. (8) and (13) into $(4)_{2}$ and $(4)_{3}$ gives

$$
\begin{aligned}
N_{2 n^{\prime}+1} \sum_{n=0}^{\infty} & A_{2 n} z_{2 n}^{(1)}(k b, k a) \bar{P}_{2 n, 2 n^{\prime}+1} \\
& +A_{2 n^{\prime}+1} z_{2 n^{\prime}+1}^{(1)}(k b, k a)-C_{n^{\prime}} h_{2 n^{\prime}+1}^{(1)}(k b) \\
= & i \varrho_{0} c v_{0} N_{2 n^{\prime}+1} \bar{P}_{0,2 n^{\prime}+1} \frac{h_{0}^{(1)}(k b)}{h_{0}^{(1) \prime}(k a)},
\end{aligned}
$$

$$
\begin{gathered}
A_{2 n^{\prime}} z_{2 n^{\prime}}^{(1) \prime}(k b, k a)-\frac{1}{2} N_{2 n^{\prime}} \sum_{n=0}^{\infty} C_{n} h_{2 n+1}^{(1) \prime}(k b) \bar{P}_{2 n^{\prime}, 2 n+1} \\
=\delta_{0, n^{\prime}} \mathrm{i} \varrho_{0} c v_{0} \frac{h_{0}^{(1) \prime}(k b)}{h_{0}^{(1) \prime}(k a)}, \\
A_{2 n^{\prime}+1} z_{2 n^{\prime}+1}^{(1) \prime}(k b, k a)-\frac{1}{2} C_{n^{\prime}} h_{2 n^{\prime}+1}^{(1) \prime}(k b)=0 .
\end{gathered}
$$

Solving numerically the above system of algebraic equations gives $A_{n}$ and $C_{n}$.

\subsection{Acoustically soft-hard boundaries}

In the case of acoustically soft-hard mixed boundaries (cf. Fig. 2d), the boundary conditions $(4)_{1},(12)_{1}$, $(12)_{2}$, and $(4)_{4}$ are satisfied together with the radiation condition (5). The acoustic pressure can be expressed by Eqs. $(7)_{2}$ and (8). Inserting Eqs. $(7)_{2}$ and (8) into $(12)_{1}$ and $(12)_{2}$ gives

$$
\begin{aligned}
& A_{2 n^{\prime}} z_{2 n^{\prime}}^{(1)}(k b, k a)-\frac{1}{2} C_{n^{\prime}} h_{2 n^{\prime}}^{(1)}(k b) \\
& =\delta_{0, n^{\prime}} \mathrm{i} \varrho_{0} c v_{0} \frac{h_{0}^{(1)}(k b)}{h_{0}^{(1) \prime}(k a)}, \\
& A_{2 n^{\prime}+1} z_{2 n^{\prime}+1}^{(1)}(k b, k a)-\frac{1}{2} N_{2 n^{\prime}+1} \sum_{n=0}^{\infty} C_{n} h_{2 n}^{(1)}(k b) \\
& \text { - } \bar{P}_{2 n, 2 n^{\prime}+1}=0 \text {, } \\
& A_{2 n^{\prime}} z_{2 n^{\prime}}^{(1) \prime}(k b, k a)+N_{2 n^{\prime}} \sum_{n=0}^{\infty} A_{2 n+1} z_{2 n+1}^{(1) \prime}(k b, k a) \\
& \cdot \bar{P}_{2 n^{\prime}, 2 n+1}-C_{n^{\prime}} h_{2 n^{\prime}}^{(1) \prime}(k b) \\
& =\delta_{0, n^{\prime}} \mathrm{i} \varrho_{0} c v_{0} \frac{h_{0}^{(1) \prime}(k b)}{h_{0}^{(1) \prime}(k a)} \text {. }
\end{aligned}
$$

Solving numerically the above system of algebraic equations gives $A_{n}$ and $C_{n}$.

\section{Acoustic radiation impedance}

The normalized time-averaged axisymmetric acoustic radiation impedance of a pulsating sphere of radius $a$ can be calculated using the Foldy's formula (FOLDY, 1949; Hasheminejad, Azarpeyvand, 2004; ThompSON Jr., 1973; 1976)

$$
\Psi=\Theta-\mathrm{i} \Xi=\frac{\int_{0}^{\pi} p(a, \theta) v_{r}^{*}(a, \theta) \sin \theta \mathrm{d} \theta}{\varrho_{0} c\left\langle\left|v_{r}(a, \theta)\right|^{2}\right\rangle},
$$

where $\Theta=\operatorname{Re} \Psi$ is the radiation resistance and $\Xi=$ $-\operatorname{Im} \Psi$ is the reactance, ${ }^{*}$ is the conjugate value, and

$$
\left\langle\left|v_{r}(a, \theta)\right|^{2}\right\rangle=\frac{1}{S} \int_{S}\left|v_{r}(a, \theta)\right|^{2} \mathrm{~d} S
$$

is the axisymmetric mean square vibration velocity on the sphere.

\subsection{Acoustically hard boundaries}

Equation (20) can be rewritten as

$$
\Psi=2 \Psi_{0}-\frac{2 \mathrm{i}}{(k a)^{2} \varrho_{0} c v_{0}} \frac{A_{0}}{h_{0}^{(1) '}(k a)},
$$


assuming that $v_{r}^{*}(a, \theta) \equiv v_{0}^{*}$, since

$$
\begin{aligned}
p(a, \theta) \equiv p_{1}(a, \theta)= & -\mathrm{i} \varrho_{0} c v_{0} \frac{h_{0}^{(1)}(k a)}{h_{0}^{(1) \prime}(k a)} \\
& +\frac{\mathrm{i}}{(k a)^{2}} \sum_{n=0}^{\infty} A_{n} \frac{P_{n}(\cos \theta)}{h_{n}^{(1) \prime}(k a)},
\end{aligned}
$$

where

$$
\Psi_{0}=\mathrm{i} \frac{h_{0}^{(1)}(k a)}{h_{0}^{(1) \prime}(k a)}=\frac{(k a)^{2}-\mathrm{i} k a}{1+(k a)^{2}},
$$

is the acoustic radiation impedance of a pulsating semisphere embedded in an infinitely extensive acoustically hard screen and radiating acoustic waves into the halfspace (obviously, this is equivalent to the acoustic radiation impedance of a pulsating sphere in free space, cf. also Jones (1986); SkUdrZYK, (1971)). The coefficients $A_{n}$ are obtained by solving numerically the system of algebraic equations in Eqs. (10) and using Eqs. (11).

The acoustic radiation resistance can also be calculated by integrating over an arbitrarily selected surface of radius $a \leqslant R \leqslant b$ as $\theta$ ranges from 0 to $\pi$ using the expression for the acoustic pressure $p_{1}$ and its radial derivative. For this purpose, we use the formula $\Theta_{1}=\operatorname{Re} \Psi_{1}$, where

$$
\Psi_{1}=\frac{R^{2}}{\varrho_{0} c\left|v_{0}\right|^{2} a^{2}} \int_{0}^{\pi} p_{1}(R, \theta) v_{1, r}^{*}(R, \theta) \sin \theta \mathrm{d} \theta
$$

and for the time harmonic vibrations

$$
v_{r}=\frac{-\mathrm{i}}{k \varrho_{0} c} \frac{\partial p}{\partial r} .
$$

We use Eq. (8) and differentiate it with respect to the radial variable $r$, obtaining

$$
\begin{aligned}
v_{1, r}^{*}(R, \theta)= & -v_{0}^{*} \frac{h_{0}^{(2) \prime}(k r)}{h_{0}^{(2) \prime}(k a)}+\frac{\mathrm{i}}{\varrho_{0} c} \sum_{n^{\prime}=0}^{\infty} \\
& \cdot A_{n^{\prime}}^{*} z_{n^{\prime}}^{(2) \prime}(k r, k a) P_{n^{\prime}}(\cos \theta),
\end{aligned}
$$

where $h^{(2)}$ is the spherical Hankel function of the second kind. We insert the above equation into (25), and obtain

$$
\begin{aligned}
\Psi_{1}= & 2 \mathrm{i} \frac{R^{2}}{a^{2}} \frac{h_{0}^{(1)}(k R) h_{0}^{(2) \prime}(k R)}{h_{0}^{(1) \prime}(k a) h_{0}^{(2) \prime}(k a)} \\
& +\frac{2}{\varrho_{0} c} \frac{R^{2}}{a^{2}}\left[\frac{A_{0}^{*}}{v_{0}^{*}} z_{0}^{(2) \prime}(k R, k a) \frac{h_{0}^{(1)}(k R)}{h_{0}^{(1) \prime}(k a)}\right. \\
& \left.-\frac{A_{0}}{v_{0}} z_{0}^{(1)}(k R, k a) \frac{h_{0}^{(2) \prime}(k R)}{h_{0}^{(2) \prime}(k a)}\right]+\frac{2 \mathrm{i}}{\left(\varrho_{0} c\right)^{2}\left|v_{0}\right|^{2}} \frac{R^{2}}{a^{2}} \\
& \cdot \sum_{n=0}^{\infty} \frac{A_{n} A_{n}^{*}}{N_{n}} z_{n}^{(1)}(k R, k a) z_{n}^{(2) \prime}(k R, k a) .
\end{aligned}
$$

The acoustic radiation resistance can also be obtained by calculating the integral over the surface of a semi-sphere of radius $R \geqslant b$ as $\theta$ goes from 0 to $\pi / 2$ using the acoustic pressure $p_{2}$ and its radial derivative. For this purpose, we use the equation $\Theta_{2}=\operatorname{Re} \Psi_{2}$, where

$$
\Psi_{2}=\frac{R^{2}}{\varrho_{0} c\left|v_{0}\right|^{2} a^{2}} \int_{0}^{\pi / 2} p_{2}(R, \theta) v_{2, r}^{*}(R, \theta) \sin \theta \mathrm{d} \theta .
$$

We use Eq. $(7)_{2}$, differentiate it with respect to $r$, and obtain

$$
v_{2, r}^{*}(R, \theta)=\frac{\mathrm{i}}{c \varrho_{0}} \sum_{n^{\prime}=0}^{\infty} C_{n^{\prime}}^{*} h_{2 n^{\prime}}^{(2) \prime}(k R) P_{2 n^{\prime}}(\cos \theta) .
$$

We insert this equation into Eq. (25) and use the orthogonality in Eq. $(38)_{2}$, and obtain

$$
\Psi_{2}=\frac{\mathrm{i}}{\left(\varrho_{0} c\right)^{2}\left|v_{0}\right|^{2}} \frac{R^{2}}{a^{2}} \sum_{n=0}^{\infty} \frac{C_{n} C_{n}^{*}}{N_{2 n}} h_{2 n}^{(1)}(k R) h_{2 n}^{(2) \prime}(k R)
$$

and the values of the coefficients $C_{n}$ are obtained by solving numerically the system of algebraic equations in Eqs. (10).

\subsection{Acoustically soft boundaries}

In the case of acoustically soft boundaries (cf. Fig. 2b), we use Eqs. (20)-(28) in a similar way as in the previous case. The main difference is that the values of the unknown coefficients $A_{n}$ and $C_{n}$ are obtained by solving numerically the system of equations in Eqs. (14) and (15) instead of (10) and (11). Next, we differentiate Eq. (7b) with respect to $r$, obtaining

$v_{2, r}^{*}(R, \theta)=\frac{\mathrm{i}}{\varrho_{0} c} \sum_{n^{\prime}=0}^{\infty} C_{n^{\prime}}^{*} h_{2 n^{\prime}+1}^{(2) \prime}(k R) P_{2 n^{\prime}+1}(\cos \theta)$

and use Eq. (29). Then the acoustic radiation resistance assumes the form $\operatorname{Re} \Psi_{2}$, where

$$
\begin{aligned}
\Psi_{2}= & \frac{\mathrm{i}}{\left(\varrho_{0} c\right)^{2}\left|v_{0}\right|^{2}} \frac{R^{2}}{a^{2}} \sum_{n=0}^{\infty} \frac{C_{n} C_{n}^{*}}{N_{2 n+1}} \\
& \cdot h_{2 n+1}^{(1)}(k R) h_{2 n+1}^{(2) \prime}(k R) .
\end{aligned}
$$

\subsection{Acoustically hard-soft boundaries}

In the case of acoustically hard-soft boundaries (cf. Fig. 2c), we use Eqs. (20)-(29) and (33) similarly to the previous cases. The main difference is that the values of the unknown coefficients $A_{n}$ and $C_{n}$ are obtained by solving numerically the system of equations in Eqs. (16) and (17) instead of (10) and (11). 


\subsection{Acoustically soft-hard boundaries}

In the case of acoustically soft-hard boundaries (cf. Fig. 2d), we use Eqs. (20)-(29) and (31) similarly to the previous cases. The main difference is that the values of the unknown coefficients $A_{n}$ and $C_{n}$ are obtained by solving numerically the system of equations in Eqs. (18) and (19) instead of (10) and (11).

\section{Illustration of the theory}

The following parameter values have been selected arbitrarily for numerical analysis. The rest density of water is $\varrho_{0}=997 \mathrm{~kg} / \mathrm{m}^{3}$. The speed of sound is $c=1497 \mathrm{~m} / \mathrm{s}$. The radius of the pulsating rigid sphere is $a=0.01 \mathrm{~m}$, its vibration velocity amplitude is $v_{0}=10^{-8} \mathrm{~m} / \mathrm{s}$, and the radius of the cavity is $b=0.02 \mathrm{~m}$ (if not stated differently). Also the angular frequency $\omega$ (in $\mathrm{rad} / \mathrm{s}$ ) has been used in the entire theoretical analysis, while the frequency $f$ (in $\mathrm{Hz}$ ) is used in the numerical analysis for practical reasons, where both quantities are related by the scaling factor $2 \pi$, i.e. $\omega=2 \pi f$. The normalized acoustic radiation impedance according to Eq. (22) is presented in Fig. 3 for the four considered boundary configurations. The radiation resistance and reactance show significant similarities for identical boundary conditions satisfied

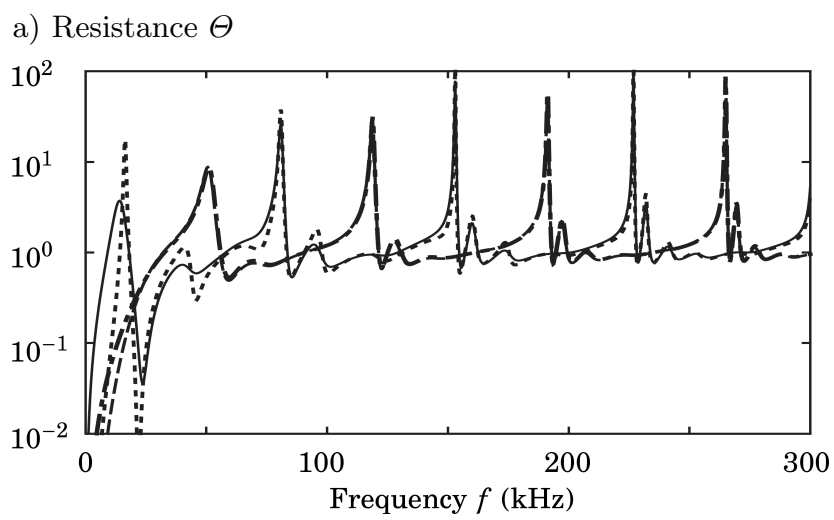

b) Reactance $\Xi$

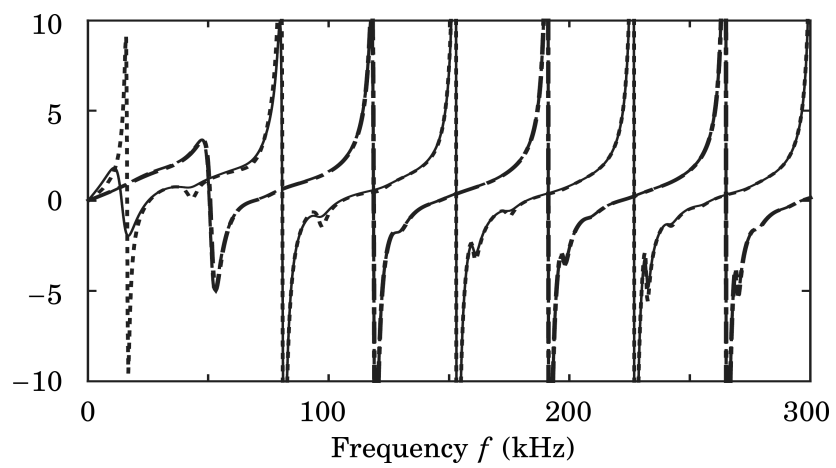

Fig. 3. Normalized acoustic impedance $\Psi=\Theta-\mathrm{i} \Xi$ of radiation and $b / a=2.0$ : a) resistance; b) reactance. Key of lines used for different boundaries: solid - hard-hard; dashed soft-soft; dotted - hard-soft; dot-dashed - soft-hard. at the cavity surface. For example, if the surface is acoustically hard, then the corresponding curves almost overlap each other for both screens - acoustically hard and soft, with the exception of the lowest frequencies, i.e. below about $100 \mathrm{kHz}$. The acoustic resistance show clear resonance maxima for frequencies strongly depending on the boundary conditions satisfied at the cavity surface. For example one of the maxima occurs at $226.8 \mathrm{kHz}$ for the acoustically hard cavity, whereas it is at $265.0 \mathrm{kHz}$ for the soft cavity. It is obvious that the radiation efficiency of a vibrating system will achieve its maxima for these frequencies, whereas the acoustic radiation reactance tends to $\pm \infty$ (which is characteristic for resonators). In contrast, in the case with no annular spherical cavity, when only the semi-sphere vibrates, resonance maxima do not occur, and the corresponding curves are relatively flat (cf. Eq. (24) and Jones (1986); SkUdrzyK (1971)). The acoustic radiation resistance can be calculated by integrating the normal component of the sound intensity $p \vec{v}^{*}$ over an arbitrarily selected surface enclosing the vibrating source. Therefore, the two further formulas in Eqs. (28) and (31) have been obtained for the normalized radiation resistance by taking their real parts. Additionally, if we integrate the normal component of the sound intensity over the semi-sphere of radius $r=b$ (connecting the subregions $\Omega_{1}$ and $\Omega_{2}$ ), then we obtain the two complex values on the basis of the two different formulas mentioned above, which should be equal. By taking their relative percentage difference, we obtain a measure of the approximation error of the acoustic radiation impedance. This difference does not exceed $10^{-10} \%$ over the entire considered frequency interval. This numerical accuracy has been achieved by using the $2 N$ initial terms in Eq. (8) and $N$ in Eqs. (7b) and (13). The number

$$
N(f)=N_{1}+\text { Round }\left[\left(N_{2}-N_{1}\right) \frac{f-f_{1}}{f_{2}-f_{1}}\right]
$$

has been calculated as the function of frequency $f$, where the following values have been selected arbitrarily $f_{1}=0 \mathrm{kHz}, f_{2}=300 \mathrm{kHz}, N_{1}=40$ and $N_{2}=100$, and Round is the nearest integer. The same numbers of terms were used in all further numerical calculations, achieving a similar accuracy. It is worth noticing that with the order of the spherical Bessel and Hankel functions reaches values of 200 , which can be considered as large. Therefore, the use of the Debye's asymptotic formulas is justified. For an advanced analysis of the use of these asymptotic formulas for the truncation of series expressions for the diffraction integrals see, e.g. VAN HAVER and JANSSEN (2014).

The spatial far-field distribution of the sound pressure level (SPL in dB, Ref. $1 \mu \mathrm{Pa}$ ) is presented in Fig. 4 at a distance of $R=100 \mathrm{~m}$ from the pulsating sphere's centre, i.e. in the far field. The graphs have been prepared for two different frequencies: $226.8 \mathrm{kHz}$ 
a)

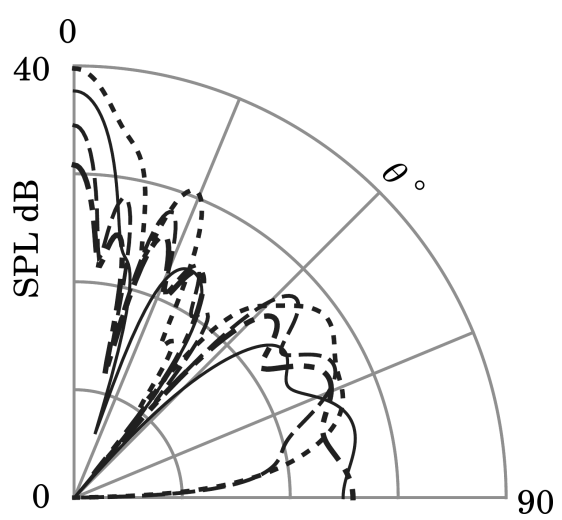

b)

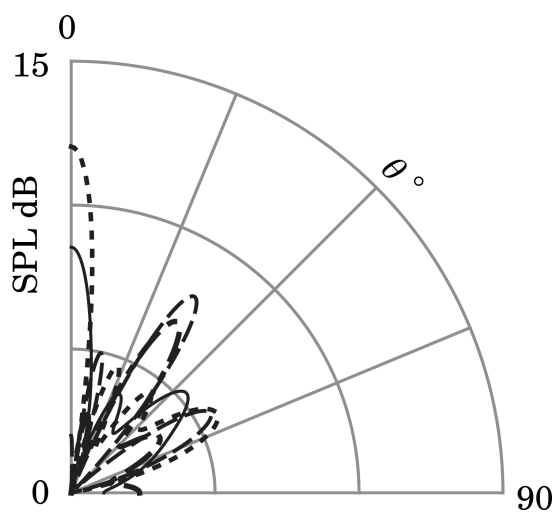

Fig. 4. Sound pressure level (SPL in $\mathrm{dB}$, Ref. $1 \mu \mathrm{Pa}$ ) in the far field for $R=100 \mathrm{~m}$ for $v_{0}=10 \mathrm{~nm} / \mathrm{s}$ and $b / a=2.0$ : a) at resonance; b) no resonance. Key of lines used for boundaries and frequency: a) solid - hard-hard at $226.8 \mathrm{kHz}$, dashed - soft-soft at $265.0 \mathrm{kHz}$, dotted - hard-soft at $226.8 \mathrm{kHz}$, dot-dashed soft-hard at $265.0 \mathrm{kHz}$; b) solid - hard-hard at $265.0 \mathrm{kHz}$, dashed - soft-soft at $226.8 \mathrm{kHz}$, dotted - hard-soft at $265.0 \mathrm{kHz}$, dot-dashed soft-hard at $226.8 \mathrm{kHz}$.

and $265.0 \mathrm{kHz}$, and for the four different boundary configurations presented in Fig. 2. These frequencies have been selected to present the cases of resonance in Fig. 4a and out of resonance in Fig. 4b. The first frequency is that of the acoustically hard cavity, and the second is that of the soft cavity. It is necessary to explain here that the same frequency $226.8 \mathrm{kHz}$ has been selected for one boundary configuration in resonance as well as for the other one in out of resonance. This if due to a simple fact that this frequency implies the resonance when the surface of the cavity is hard, and is out of resonance when the surface is soft. A similar situation occurs for the frequency $265.0 \mathrm{kHz}$, but with reversed boundary condition on the surface of the cavity. Also, when the resonator is in the resonance the frequency must be selected very accurately (see very narrow maxima for the radiation impedance around resonance in Fig. 3a), and there is no such need in out of resonance. The SPL assumes much greater values in resonance compared to the values at out of resonance. The average SPL in Fig. $4 \mathrm{a}$ is about $25 \mathrm{~dB}$ and about $7 \mathrm{~dB}$ in (4b), which gives the difference of about $18 \mathrm{~dB}$. The SPL increases up to about $38 \mathrm{~dB}$ in resonance when the deflection angle from the main direction tends to zero $(\theta \rightarrow 0)$. This gives an increase of more than $10 \mathrm{~dB}$ in the main direction compared to the other directions. The effect is strongest for the hardhard boundaries. A very narrow sound beam is formed for the deflection angles smaller than about 5 degrees. In contrary, in out of resonance the SPL does not exceed $12 \mathrm{~dB}$ for all boundary configurations and for all directions.

The near-field spatial distribution of the sound pressure level is presented in Figs. 5 and 6 for distances from the pulsating sphere ranging from $1 \mathrm{~cm}$ to $6 \mathrm{~cm}$. The observations are similar to the conclusions obtained from the analysis of Fig. 4. A considerable growth in the sound pressure level in the main direction can be observed for the resonance frequencies and the four different boundary configurations (cf. Fig. 5). In contrast, the acoustic field is scattered in all direction for other frequencies (cf. Fig. 6). It is worth noting that the acoustic pressure tends to zero in a narrow layer directly adjoining all the acoustically soft boundaries. For example, they are the soft surfaces of the cavity and flat screen in Fig. 5b whereas it is only the cavity surface in Fig. 5d.

The pulsating sphere submerged in water forms a resonator together with the semi-spherical cavity. A question arises: what is the effect of the quotient of the radii of the cavity and the sphere on the radiated acoustic field? Figure 7 presents a schematic view of the resonator for three different values of this quotient. All the numerical results presented herein apply to the geometry presented in Fig. 7b. Therefore the differences will now be discussed for the resonator geometries presented in Figs. 7a and 7c. As mentioned before, the boundary conditions satisfied in the cavity have the most significant effect on the sound field. Therefore, further analysis will be limited to two boundary configurations: the hard-hard and soft-soft boundary configurations. The acoustic radiation impedance is presented in Fig. 8 for the three different geometry configurations of the resonator presented in Fig. 7. It can be seen that the first resonance frequency differs slightly for the three geometries, and is equal to about $15 \mathrm{kHz}$ for the hard-hard boundaries. However, the differences are much more significant for the second resonance frequency, which is about $155 \mathrm{kHz}$ for $b / a=1.5,80 \mathrm{kHz}$ for $b / a=2.0$ (see Fig. 3), and $55 \mathrm{kHz}$ for $b / a=2.5$. The differences are even greater for all the higher resonance frequencies. The situation is similar for the softsoft boundaries, with the difference that now even for 
a)

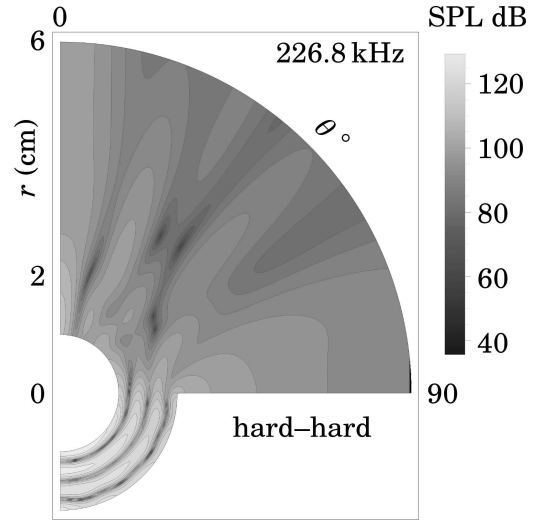

c) 6

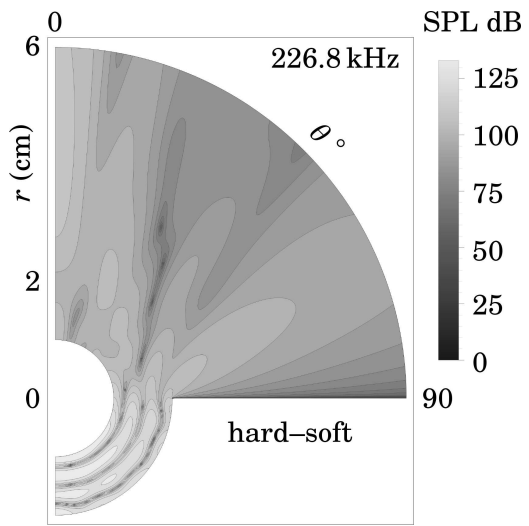

b) 6

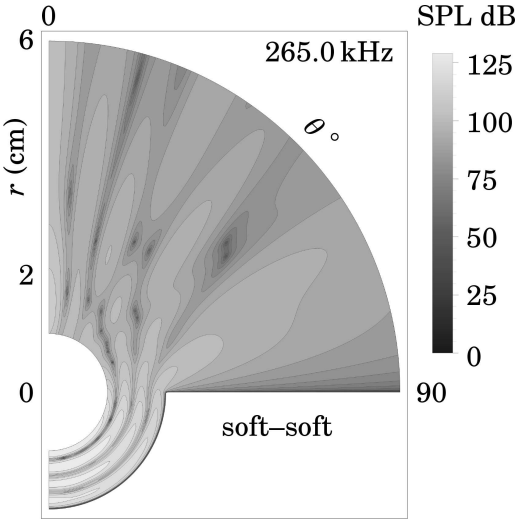

d)

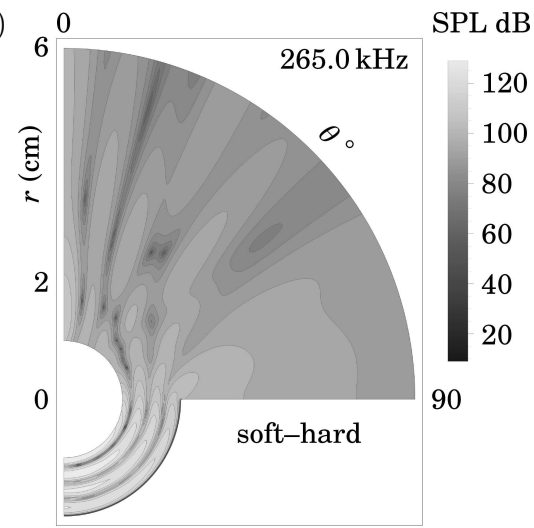

Fig. 5. Sound pressure level in resonance (SPL in $\mathrm{dB}$, Ref. $1 \mu \mathrm{Pa}$ ) for four different boundary configurations, $v_{0}=10 \mathrm{~nm} / \mathrm{s}$ and $b / a=2.0$.

a)

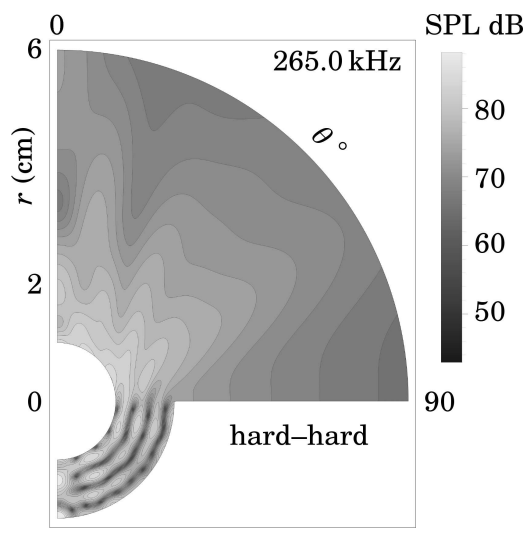

c)

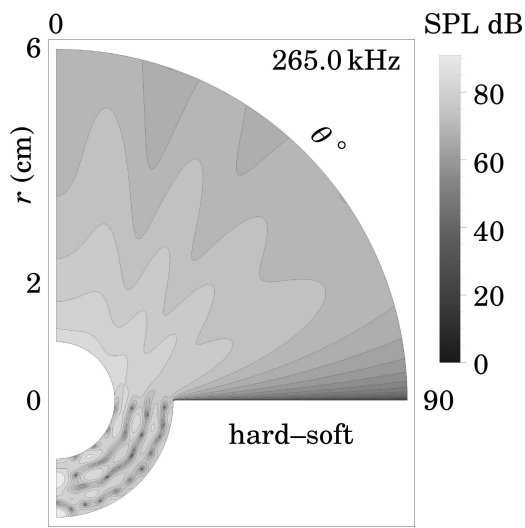

b)

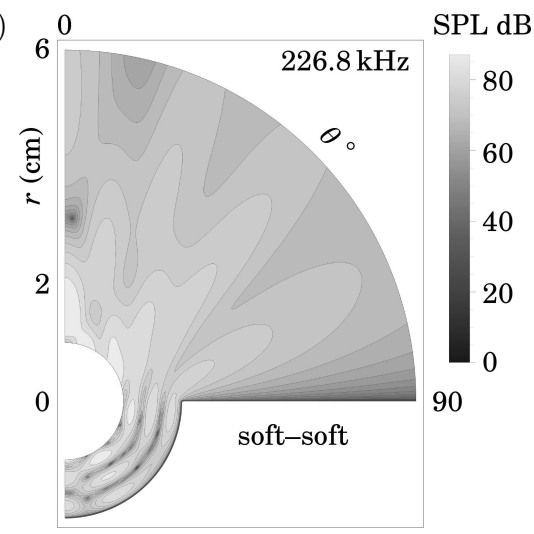

d)

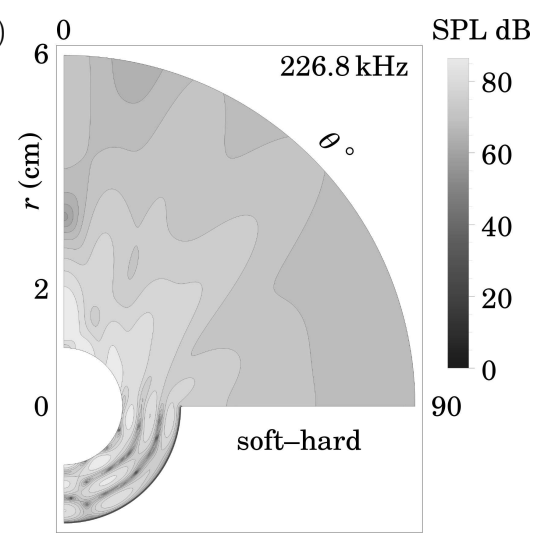

Fig. 6. Sound pressure level outside resonance (SPL in dB, Ref. $1 \mu \mathrm{Pa}$ ) for four different boundary configurations, $v_{0}=10 \mathrm{~nm} / \mathrm{s}$ and $b / a=2.0$. 
a) $b / a=1.5$

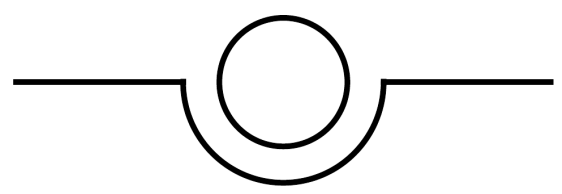

b) $b / a=2.0$

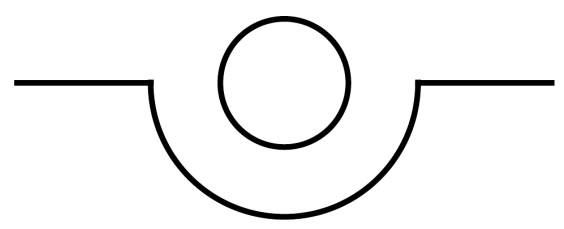

c) $b / a=2.5$

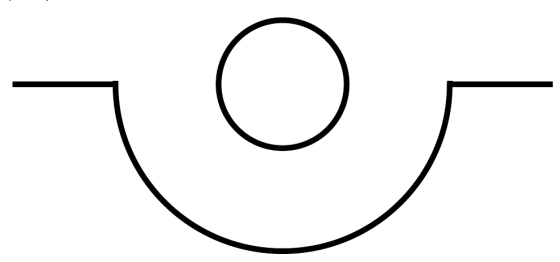

Fig. 7. Three different ratios of cavity and sphere $b / a$.

a) Resistance $\Theta$ (hard-hard)

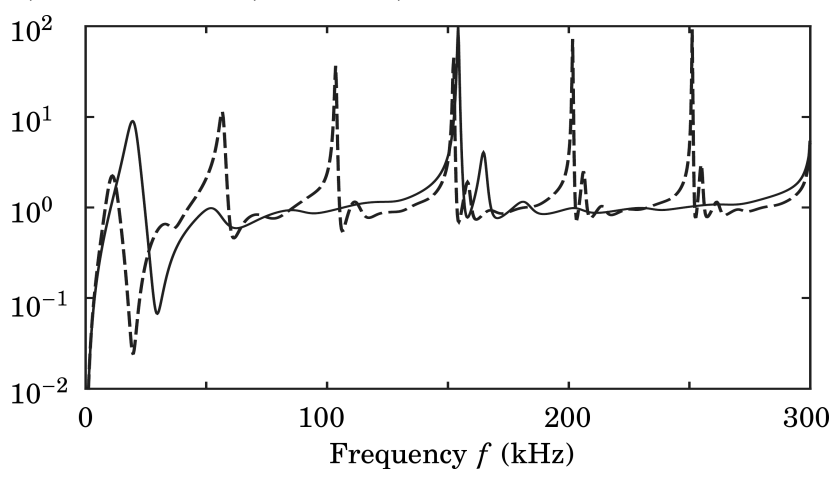

b) Resistane $\Theta$ (soft-soft)

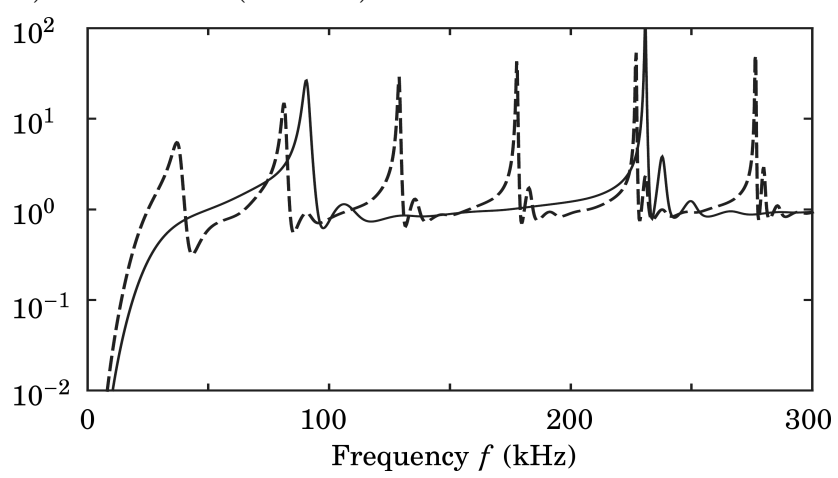

Fig. 8. Normalized acoustic resistance $\Theta$ of radiation for the two different boundary configurations. Key of lines used for different values of cavity geometry parameter $b / a$ : solid 1.5 ; dashed -2.5 (for $b / a=2.0$ see Fig. 3 ).

the first resonance frequency the differences are significant, since it is about $90 \mathrm{kHz}$ for $b / a=1.5,50 \mathrm{kHz}$ for $b / a=2.0$ (see Fig. 3), and $40 \mathrm{kHz}$ for $b / a=2.5$. The eigenfrequencies of a circular spherical cavity were investigated by FERRIS (1952), FLANAGAN (1965), and Russell (20010). They applied the boundary conditions to the general solution for the acoustic potential inside the cavity. A similar approach can be used to determine the eigenfrequencies of an annular spherical cavity. The frequency equations for hard and soft surfaces of radius $b$ of the cavity (surface of the sphere of radius $a$ is hard in both cases) can be obtained easily by applying the boundary conditions $\partial p /\left.\partial r\right|_{r=a}=0$, and $\partial p /\left.\partial r\right|_{r=b}=0$ and $\left.p\right|_{r=b}=0$, respectively, to the general eigenfunction given by MORSE (1948)

$$
\widetilde{A}_{n} j_{n}(k r)+\widetilde{B}_{n} y_{n}(k r)
$$

and achieving the frequency equations

$$
\begin{aligned}
& j_{n}^{\prime}(\beta) y_{n}^{\prime}(s \beta)-j_{n}^{\prime}(s \beta) y_{n}^{\prime}(\beta)=0, \\
& j_{n}^{\prime}(\beta) y_{n}(s \beta)-j_{n}(s \beta) y_{n}^{\prime}(\beta)=0,
\end{aligned}
$$

respectively, where $s=b / a$ is the geometric factor of the cavity, and $\beta=k a$ is the eigenvalue. The initial eigenfrequencies are presented in Table 1 . Determining the constants $\widetilde{A}_{n}$ and $\widetilde{B}_{n}$ is necessary to determine the acoustic potential within the cavity, but neither for obtaining the frequency equations nor eigenfrequencies. The relation between the eigenvalue and the eigenfrequency is $f_{\ell}^{n}=\beta_{\ell}^{n} c / 2 \pi a$, where $n=0,1,2, \ldots, \infty$ is the order of the spherical functions and $\ell=1,2,3, \ldots, \infty$ is the number of the successive eigenfrequency. Bearing in mind that these eigenfrequencies apply to the full cavity, i.e. when the boundary conditions are satisfied for $0 \leqslant \theta \leqslant \pi$, we can try to assign them to the resonance frequencies of the semi-spherical cavity considered herein. It can be noticed that the resonance frequency $226.8 \mathrm{kHz}$ falls between the eigenfrequencies $f_{4}^{0}$ and $f_{4}^{1}$ (obtained from Eq. $\left.(36)_{1}\right)$ for the hard surface of radius $b$ of the cavity and for $b / a=2.0$, while the very frequency results from the superposition of the eigenfunctions (35) and is controlled by the constants $\widetilde{A}_{n}$ and $\widetilde{B}_{n}$. For the soft surface of radius $b$ of the cavity and for $b / a=2.0$, the resonance frequency $265.0 \mathrm{kHz}$ falls also between the eigenfrequencies $f_{4}^{0}$ and $f_{4}^{1}$ (obtained from Eq. $(36)_{2}$ ). The lowest resonance frequency falls alway between the eigenfrequencies $f_{1}^{0}$ and $f_{1}^{1}$ in the presented study. It can be noted that the lowest and the successive eigenfrequencies increases when the ratio $b / a$ decreases. The effect of the resonator geometry on the resonance frequencies is significant (cf. Table 1). Additional evidence for this conclusion can be found in Figs. 9 and 10. The far-field sound pressure level is presented in Fig. 9. The frequencies have been selected for resonance when the cavity geometry parameter $b / a$ is equal to 2.0, as in Fig. 4a, i.e. $226.8 \mathrm{kHz}$ for the hard cavity and $265.0 \mathrm{kHz}$ for the soft one. However, the parameter is changed in Fig. 9 and assumes the values of 
Table 1. The initial eigenfrequencies $f_{\ell}^{n}$ (in $\mathrm{kHz}$ ) of a annular spherical cavity of a fixed inner radius $a=1 \mathrm{~cm}$ and the outer cavity surface of radius $b=s a$ being hard or soft (b.c - boundary conditions).

\begin{tabular}{|c|c|c|c|c|c|c|c|c|c|}
\hline b.c. & $s$ & $n$ & $\ell=1$ & $\ell=2$ & $\ell=3$ & $\ell=4$ & $\ell=5$ & $\ell=6$ & $\ell=7$ \\
\hline hard & 1.5 & 0 & 0.000 & 152.145 & 300.653 & 449.939 & 599.431 & 749.005 & 898.621 \\
hard & 1.5 & 1 & 26.755 & 154.748 & 301.929 & 450.786 & 600.064 & 749.511 & 899.043 \\
soft & 1.5 & 0 & 87.516 & 229.480 & 377.255 & 526.106 & 675.330 & 824.726 & 974.215 \\
soft & 1.5 & 1 & 92.323 & 231.172 & 378.269 & 526.830 & 675.893 & 825.186 & 974.604 \\
hard & 2.0 & 0 & 0.000 & 78.291 & 151.546 & 225.799 & 300.342 & 375.005 & 449.730 \\
hard & 2.0 & 1 & 21.923 & 82.335 & 153.499 & 227.081 & 301.298 & 375.768 & 450.365 \\
soft & 2.0 & 0 & 48.336 & 117.059 & 190.096 & 264.118 & 338.499 & 413.048 & 487.688 \\
soft & 2.0 & 1 & 54.915 & 119.638 & 191.630 & 265.209 & 339.345 & 413.739 & 488.273 \\
hard & 2.5 & 0 & 0.000 & 53.741 & 101.966 & 151.183 & 200.723 & 250.403 & 300.154 \\
hard & 2.5 & 1 & 18.398 & 58.648 & 104.370 & 152.746 & 201.881 & 251.324 & 300.919 \\
soft & 2.5 & 0 & 34.541 & 79.476 & 127.680 & 176.778 & 226.217 & 275.819 & 375.257 \\
soft & 2.5 & 1 & 41.663 & 82.637 & 129.552 & 178.100 & 227.239 & 276.652 & 375.866 \\
\hline
\end{tabular}

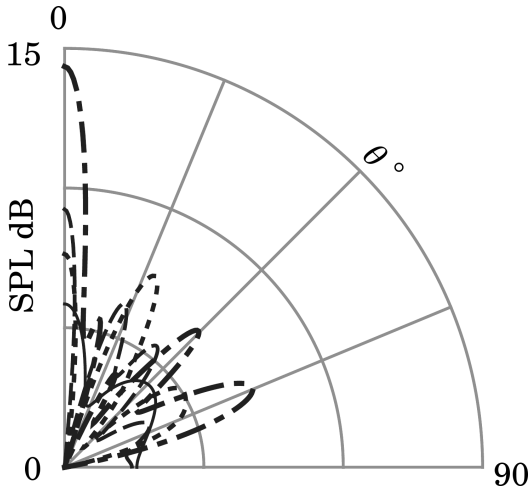

a)

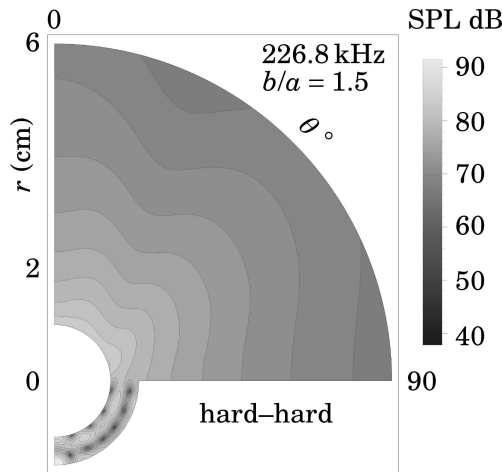

b)

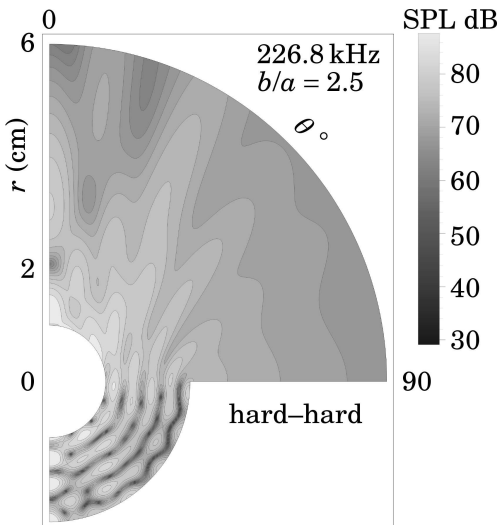

Fig. 9. Sound pressure level (SPL in dB, Ref. $1 \mu \mathrm{Pa}$ ) in the far field for $R=100 \mathrm{~m}$ for $v_{0}=10 \mathrm{~nm} / \mathrm{s}$. Key of lines used for different boundaries, frequencies, and values of cavity geometry parameter: solid - hard-hard at $226.8 \mathrm{kHz}, b / a=1.5$; dashed - hard-hard at $226.8 \mathrm{kHz}$, $b / a=2.5$; dotted - soft-soft at $265.0 \mathrm{kHz}, b / a=1.5$; dot-dashed - soft-soft at $265.0 \mathrm{kHz}, b / a=2.5$.

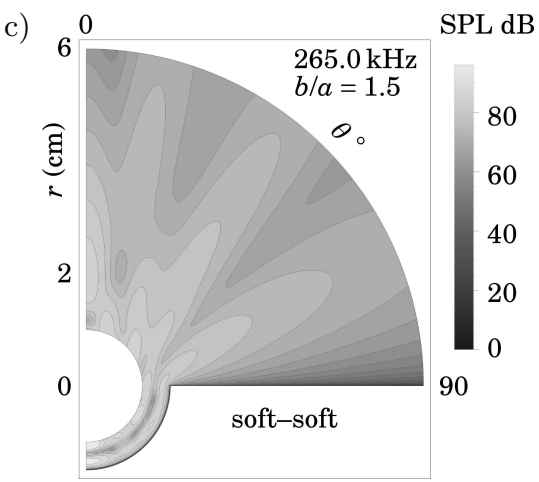

d)

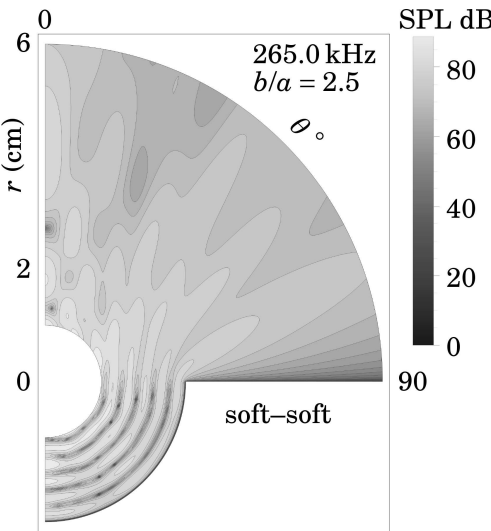

Fig. 10. Sound pressure level in resonance (SPL in dB, Ref. $1 \mu \mathrm{Pa}$ ) for $v_{0}=10 \mathrm{~nm} / \mathrm{s}$ and $b / a=1.5$ and 2.5 . The graphs for sound pressure level in resonance and for the same boundaries and $b / a=2.0$ are presented earlier in Figs. $5 \mathrm{a}$ and $5 \mathrm{~b}$. 
1.5 and 2.5, and the fluid vibrating in the cavity is out resonance (cf. Fig. 8). Consequently, the SPL assumes values smaller than $15 \mathrm{~dB}$ in the main direction and smaller than $8 \mathrm{~dB}$ in all the other directions. Figure 10 is a completion of Figs. $5 \mathrm{a}$ and $5 \mathrm{~b}$ for $b / a$ equal to 1.5 and 2.5. The SPL values are about by $25 \mathrm{~dB}$ smaller than in resonance in Fig. 5, and do not exceed $100 \mathrm{~dB}$ in any of the near-fields.

\section{Concluding remarks}

A rigorous analysis of the acoustic radiation impedance of the resonator submerged in water enabled determining the resonance frequencies. Consequently, we obtain a highly effective source of a very narrow acoustic beam for these frequencies. It has been found that the quotient of the radii of the cavity and the pulsating sphere has a significant effect, especially on the higher-order resonance frequencies. The method presented enables determining the acoustic near- and far-field. The formulas for the acoustic pressure and acoustic radiation impedance contain series of high numerical accuracy and efficiency, which has been examined empirically. The results presented herein probably can be useful for designing highly efficient hydroacoustic transducers. This is, however, a subject for a further experimental analysis.

\section{Appendix. A useful formula}

The following relation can be useful (cf. Abramowitz and Stegun (1972, Eqs. 8.6.1 and 8.6.3) assuming $\mu=0$ and $\nu=2 n+2$ and $2 n+1$, respectively)

$$
\begin{aligned}
\left.\frac{\mathrm{d}}{\mathrm{d} \theta} P_{2 n+1}(\cos \theta)\right|_{\theta=\pi / 2} & =(2 n+2) P_{2 n+2}(0) \\
& =2(-1)^{n+1} \frac{\Gamma(n+3 / 2)}{\sqrt{\pi} \Gamma(n+1)}
\end{aligned}
$$

where $n=0,1,2, \ldots, P_{2 n+1}(0)=0$, and $P_{0}(\cos \theta)=1$.

The orthogonality of the Legendre polynomials can be presented as (ABRAmowitz and Stegun (1972, p. 338, Eqs. 8.14.10 and 8.14.13) and Gradshteyn and RYZHIK (2007, p. 769, Eq. 7.113.1)

$$
\begin{aligned}
\int_{0}^{\pi} P_{n}(\cos \theta) P_{n^{\prime}}(\cos \theta) \sin \theta \mathrm{d} \theta & =\frac{2}{N_{n}} \delta_{n n^{\prime}}, \\
\int_{0}^{\pi / 2} P_{2 n}(\cos \theta) P_{2 n^{\prime}}(\cos \theta) \sin \theta \mathrm{d} \theta & =\frac{1}{N_{2 n}} \delta_{n n^{\prime}}, \\
\int_{0}^{\pi / 2} P_{2 n}(\cos \theta) P_{2 n^{\prime}+1}(\cos \theta) \sin \theta \mathrm{d} \theta & =\bar{P}_{2 n, 2 n^{\prime}+1}
\end{aligned}
$$

for $m, n, n^{\prime}=0,1,2, \ldots$, where

$$
\begin{aligned}
N_{\nu} & =2 \nu+1 \\
\bar{P}_{2 n, 2 n^{\prime}+1} & =\frac{(-4)^{-n-n^{\prime}-1}(2 n) !\left(2 n^{\prime}+1\right) !}{\left(n-n^{\prime}-1 / 2\right)\left(n+n^{\prime}+1\right)(n !)^{2}\left(n^{\prime} !\right)^{2}} .
\end{aligned}
$$

\section{Acknowledgments}

The research presented in this paper was partially supported under The Centre for Innovation and Transfer of Natural Sciences and Engineering Knowledge Project at The University of Rzeszów in Poland.

\section{References}

1. Aarts R.M., Janssen A.J.E.M. (2010), Sound radiation from a resilient spherical cap on a rigid sphere, Journal of the Acoustical Society of America, 127, 4, 2262-2273, doi: 10.1121/1.3303978.

2. Aarts R.M., Janssen A.J.E.M. (2011), Comparing sound radiation from a loudspeaker with that from a flexible spherical cap on a rigid sphere, AES: Journal of the Audio Engineering Society, 59, 4, 201-212.

3. Abramowitz M., Stegun I.A. [Eds.] (1972), Handbook of Mathematical Functions with Formulas, Graphs, and Mathematical Tables, U.S. Department of Commerce, National Bureau of Standards.

4. Anderson V.C. (1950), Sound scattering from a fluid sphere, Journal of the Acoustical Society of America, 22, 4, 426-431, doi: 10.1121/1.1906621.

5. Azarpeyvand M. (2005), Active noise cancellation of a spherical multipole source using a radially vibrating spherical baffled piston, Acoustical Physics, 51, 6, 609618, doi: 10.1134/1.2130891.

6. Azarpeyvand M. (2014), Prediction of negative radiation forces due to a bessel beam, Journal of the Acoustical Society of America, 136, 2, 547-555, doi: 10.1121/1.4884758.

7. Azarpeyvand M., Azarpeyvand M. (2014), Application of acoustic bessel beams for handling of hollow porous spheres, Ultrasound in Medicine and Biology, 40, 2, 422-433, doi: 10.1016/j.ultrasmedbio.2013.07.008.

8. Barmatz M., Collas P. (1985), Acoustic radiation potential on a sphere in plane, cylindrical, and spherical standing wave fields, Journal of the Acoustical Society of America, 77, 3, 928-945, doi: 10.1121/1.392061.

9. Brański A., Leniowska L. (1992), Far field of a concentric ring vibrating with constant velocity on a rigid sphere flow over side branch deep cavity in a rectangular duct, Archives of Acoustics, 17, 2, 277286, http://acoustics.ippt.pan.pl/index.php/aa/article/ view/1188.

10. FARAN J.J. (1951), Sound scattering by solid cylinders and spheres, The Journal of the Acoustical Society of America, 23, 4, 405-418, doi: 10.1121/1.1906780. 
11. FERris H.G. (1952), The free vibrations of a gas contained within a spherical vessel, The Journal of the Acoustical Society of America, 24, 1, 57-60, doi: $10.1121 / 1.1906848$.

12. Flanagan J.L. (1965), Acoustic modes of a hemispherical room, The Journal of the Acoustical Society of America, 37, 4, 616-618, doi: 10.1121/1.1909379.

13. Foldy Leslie L. (1949), Theory of passive linear electroacoustic transducers with fixed velocity distribution, The Journal of the Acoustical Society of America, 21, 6, 595-604, doi: 10.1121/1.1906556.

14. Gaunaurd G.C., Huang H. (1996), Sound scattering by a spherical object near a hard flat bottom, IEEE Transactions on Ultrasonics, Ferroelectrics, and Frequency Control, 43, 4, 690-700, doi: $10.1109 / 58.503731$.

15. Gradshteyn I.S., Ryzhik I.M. (2007), Table of Integrals, Series, and Products, Academic Press, New York, 7 edition.

16. Hasheminejad S.M. (2003), Modal acoustic impedance force on a spherical source near a rigid interface, Acta Mechanica Sinica, 19, 1, 33-39, doi: 10.1007/BF02487450.

17. Hasheminejad S.M., Azarpeyvand M. (2003a), Eccentricity effects on acoustic radiation from a spherical source suspended within a thermoviscous fluid sphere, IEEE Transactions on Ultrasonics, Ferroelectrics, and Frequency Control, 50, 11, 1444-1454, doi: 10.1109/TUFFC.2003.1251128.

18. Hasheminejad S.M., Azarpeyvand M. (2003b), Non-axisymmetric acoustic radiation from a transversely oscillating rigid sphere above a rigid/compliant planar boundary, Acta Acustica united with Acustica, 89, 6, 998-1007.

19. Hasheminejad S.M., Azarpeyvand M. (2004a), Acoustic radiation from a pulsating spherical cap set on a spherical baffle near a hard/soft flat surface, IEEE Journal of Oceanic Engineering, 29, 1, 110-117, doi: 10.1109/JOE.2003.822978.

20. Hasheminejad S.M., Azarpeyvand M. (2004b), Sound radiation due to modal vibrations of a spherical source in an acoustic quarterspace, Shock and Vibration, 11, 5-6, 625-635.

21. Jeong W.T., Kang Y.J., Kim S.H. (2012), Acoustic transmission analysis on cavity resonance sound in a cylindrical cavity system: Application to a korean bell, Journal of the Acoustical Society of America, 131, 2, 1547-1557, doi: 10.1121/1.3675552.

22. Jones D.S. (1986), Acoustic and Electromagnetic Waves, Clarendon Press, Oxford.

23. Kim K., Lauchle G.C., Gabrielson T.B. (2008), Near-field acoustic intensity measurements using an accelerometer-based underwater intensity vector sensor, Journal of Sound and Vibration, 309, 1-2, 293306, doi: 10.1016/j.jsv.2007.07.024.
24. Kolber K., Snakowska A., Kozupa M. (2014), The effect of plate discretization on accuracy of the sound radiation efficiency measurements, Archives of Acoustics, 39, 4, 511-518, doi: 10.2478/aoa-2014-0055.

25. Levine H. (2001), Acoustical cavity excitation, Journal of the Acoustical Society of America, 109, 6, 25552565, doi: 10.1121/1.1367246.

26. Levine H., Leppington F.G. (1991), The acoustic power from moving and pulsating spheres, Journal of Sound and Vibration, 146, 2, 199-210, doi: 10.1016/0022-460X(91)90759-D.

27. Morse P.M. (1948), Vibration and Sound, McGrawHill, New York, 2 edition.

28. Pelat A., Félix S., Pagneux V. (2009), On the use of leaky modes in open waveguides for the sound propagation modeling in street canyons, Journal of the Acoustical Society of America, 126, 6, 2864-2872, doi: 10.1121/1.3259845.

29. Rudgers A.J. (1974), A correlation technique for determining the self- and mutual-radiation impedances of transducers in an array, The Journal of the Acoustical Society of America, 55, 4, 759-765, doi: 10.1121/1.1914596.

30. Russell D.A. (2010), Basketballs as spherical acoustic cavities, American Journal of Physics, 78, 6, 549-554, doi: 10.1119/1.3290176.

31. Skudrzyk E. (1971), The Foundations of Acoustics, Basic Mathematics \& Basic Acoustics, SpringerVerlag, Wien, New York.

32. Sommerfeld A. (1964), Partial Differential Equations in Physics, volume 6 of Lectures on Theoretical Physics, Academic Press, New York.

33. Szemela K. (2015), Sound radiation inside an acoustic canyon with a surface sound source located at the bottom, Journal of Computational Acoustics, 23, 3, 1550014 (22 pages), doi: 10.1142/S0218396X15500149.

34. TAng Y.-Z., Wu Z.-J., TAng L.-G. (2010), Analysis and improvement of sound radiation performance of spherical cap radiator, Chinese Physics B, 19, 5, 0543031-0543039, doi: 10.1088/16741056/19/5/054303.

35. Thompson JR. W. (1973), Acoustic radiation from a spherical source embedded eccentrically within a fluid sphere, Journal of the Acoustical Society of America, 54, 6, 1694-1707, doi: 10.1121/1.1914469.

36. Thompson JR. W. (1976), Radiation from a spherical acoustic source near a scattering sphere, Journal of the Acoustical Society of America, 60, 4, 781-787, doi: 10.1121/1.381158.

37. Van Haver S., Janssen A.J.E.M. (2014), Truncation of the series expressions in the advanced ENZ-theory of diffraction integrals, Journal of The European Optical Society-Rapid Publications, 9, 14042 (13 pages), doi: 10.2971/jeos.2014.14042. 